\title{
A Highly Efficient Neutron Veto for Dark Matter Experiments
}

\author{
Alex Wright*, Pablo Mosteiro, Ben Loer, Frank Calaprice \\ Physics Department, Princeton University, Princeton NJ, 08544, USA
}

\begin{abstract}
We present a conceptual design for an active neutron veto, based on boron-loaded liquid scintillator, for use in direct-detection dark matter experiments. The simulated efficiency of a 1 meter thick veto, after including the effects of neutron captures in the inner detector and inefficiencies due to feed-throughs into the veto, is greater than $99.5 \%$ for background events produced by radiogenic neutrons, while the background due to externally produced cosmogenic neutrons is reduced by more than $95 \%$. The ability of the veto to both significantly suppress, and provide in situ measurements of, these two dominant sources of background would make the next generation of dark matter experiments much more robust, and dramatically improve the credibility of a dark matter detection claim based on the observation of a few recoil events. The veto would also allow direct extrapolation between the background-free operation of a small detector and the physics reach of a larger detector of similar construction.
\end{abstract}

Keywords: direct-detection dark matter search, low-background techniques, neutron veto, boron-loaded liquid scintillator

\section{Introduction}

As the sensitivity of direct detection dark matter experiments continues to improve, the suppression of neutron backgrounds through the selection of highly radiopure materials and passive shielding becomes much more difficult. Indeed, experiments are reaching sensitivities where backgrounds estimates

\footnotetext{
*Corresponding author: Department of Physics, Jadwin Hall, Princeton University, Princeton NJ, 08540, USA. Tel: (609) 258-0154 Email: ajw@princeton.edu
} 
using calculations based on ex situ measurements of representative material samples are no longer sufficiently robust, as relatively modest differences in production methods and material handling procedures can have a significant effects on the overall background in extremely pure materials. Therefore, the ability to precisely measure background levels in situ will be critical to giving future dark matter experiments the ability to make credible claims of dark matter detection.

As direct detection dark matter experiments seek to detect WIMP dark matter by observing nuclear recoils produced by WIMP interactions with nuclei, and as single scatter neutron events can produce nuclear recoils that are indistinguishable from WIMP interactions, the issue of in situ background control is particularly relevant in considering neutron backgrounds. These neutrons are produced both within the material comprising the detectors themselves and in the materials surrounding them through radiogenic processes $((\alpha, \mathrm{n})$ reactions and spontaneous fission) and by spallation by cosmicray muons. The neutron-induced background in dark matter detectors has typically been minimized by constructing the detectors from materials containing extremely low levels of radioactive contaminants, by passively shielding the experiments against externally produced radiogenic neutrons, and by operating the experiments in underground laboratories where the cosmogenic neutron rate is reduced. As mentioned above, however, these strategies, are reaching their practical limits; the size and sensitivity of dark matter experiments is reaching the point where it is extremely difficult to demonstrate, using ex-situ assay techniques, that the materials used in the construction of a given detector are sufficiently free from radioactivity that the production of a small number of neutron-induced recoil events is unlikely. In addition, only the deepest of underground laboratories have cosmogenic neutron fluxes low enough to permit the operation of next generation of dark matter experiments in conventional shielding with a robust expectation having no cosmogenically-induced background. These effects combine to both limit the sensitivity of experiments and to make it extremely difficult for any experiment to claim, with high confidence, that the detection of a few recoil events constitutes a detection of dark matter. Indeed, at the present time all of the leading dark matter experiments see some nuclear-recoil-like events, so these experiments are either background limited or unable to make a detection claim based on the few events observed.

Replacing the passive neutron shielding with an active neutron veto is one way in which experiments can both lower their neutron backgrounds and 
make precise in situ assays of this important background. A high-efficiency active neutron veto would not only significantly improve the sensitivity of an experiment which would otherwise be limited by neutron backgrounds, but it would also allow an experiment which has been successful in achieving a low neutron background (and which hence has very few observed coincidences between nuclear recoils in the dark matter detector and signals in the veto) to demonstrate convincingly that the number of neutron-induced recoil backgrounds possible in the data is extremely small. It is conceivable that with a high efficiency veto these limits could be as low as a few hundredths of an event. This would help to give experiments the ability to make convincing claims of dark matter detection based on the observation of a few events, and help to move the field from working to set better limits on the dark matter interaction cross section to attempting to detect dark matter interactions. In addition, the ability to extrapolate a carefully understood background, coupled with the direct suppression afforded by the neutron veto, would allow a smaller experiment to demonstrate directly the potential for a much larger experiment of similar construction to operate background free. This extrapolation ability would be extremely helpful in guiding the development of ever larger and more sensitive detectors. The practical utility of all of these applications, however, is dictated by the absolute efficiency of the neutron veto; as a result, the development of a highly efficient veto system is extremely desirable.

The obvious benefit of neutron veto capability has led most current dark matter experiments to implement veto procedures. In some, the veto is achieved by segmenting (through position reconstruction and/or physical segmentation) the active volume of the dark matter detector to look for neutrons that produce more than one recoil event. Other experiments [[1], [2] have deployed separate, dedicated veto systems. In the future, very large, monolithic detectors could veto neutron events quite efficiently using internal coincidences, except near the detector walls where there is a reasonable probability for the recoiling neutron to escape, and where neutrons with only enough energy to produce a single detectable recoil are most likely to interact. In order to have a highly efficient neutron veto, then, these large detectors will have to either take a reasonably large cut in fiducial volume or install an external neutron veto.

A neutron veto system with a very high detection efficiency can be produced by surrounding a dark matter detector with a layer of liquid scintillator. Such a veto, with a thickness of order 1 meter, is sufficient to detect 
a very high percentage of the radiogenic neutrons produced by the inner detector, and a significant fraction of cosmogenic neutrons. However, at about $250 \mu \mathrm{s}$, the capture time for thermal neutrons in a liquid scintillator is rather long. This means that in order to efficiently veto the (promptly produced) neutron-induced dark matter backgrounds, veto windows of millisecond duration are necessary. With such a long veto window, the background rate in the scintillator must be quite low, less than $\sim 100 \mathrm{~Hz}$, to keep the veto-induced dead time in the dark matter detector from becoming significant. Achieving such a low event rate in the veto requires bulky and expensive passive shielding; even the use of conventional photomultiplier tubes to instrument the veto becomes difficult, as the event rate due to radioactive contaminants in the PMTs themselves would be excessive.

In this paper we show that relatively compact, highly efficient neutron vetoes for dark matter detectors are practically realizable through the use of boron-loaded liquid scintillator. We have studied such a veto using Geant4based Monte Carlo simulations in the context of the proposed DarkSide-50 dark matter detector. The efficiency of the veto is shown to be very high, even after taking into account neutrons which do not escape the inner detector and potential inefficiencies due to feed-through penetrations. The total event rate in the veto from internal and external sources is estimated and found (with a relatively modest amount of passive shielding against external gamma-rays) to be acceptable, even using conventional PMTs. In large part, this tolerance of veto rate is due to the decrease in the neutron capture time afforded by the large capture cross-section of ${ }^{10} \mathrm{~B}$. Optical simulations of the veto show that the very low energy threshold necessary to reliably detect the reaction products of neutron capture on ${ }^{10} \mathrm{~B}$ is comfortably achievable, even under fairly conservative assumptions about the optical characteristics of the veto.

\section{Boron-Loaded Scintillator}

Neutron detection using boron-loaded liquid scintillator, produced by adding tri-methyl borate (TMB) to standard scintillator cocktails, was first investigated in [3] and subsequently developed into practical detectors by [4].

${ }^{10} \mathrm{~B}$, which has a natural abundance of about $20 \%$, captures thermal neutrons with a very high $(3837(9)$ b) total capture cross section via two channels [5]:

$$
\begin{array}{rlr}
{ }^{10} \mathrm{~B}+n & \left.\rightarrow{ }^{7} \mathrm{Li} \text { (g.s. }\right)+\alpha \\
& \rightarrow{ }^{7} \mathrm{Li}^{*}+\alpha,{ }^{7} \mathrm{Li}^{*} \rightarrow{ }^{7} \mathrm{Li}+\gamma(478 \mathrm{keV}) & 63.7 \%
\end{array}
$$


Importantly for the current discussion, the nuclear recoil reaction products carry a significant amount of energy (in the decay to the excited state of ${ }^{7} \mathrm{Li}$, $\mathrm{E}(\alpha)=1471 \mathrm{keV}$ and $\mathrm{E}\left({ }^{7} \mathrm{Li}\right)=839 \mathrm{keV}$, while for the ground state decay $\mathrm{E}(\alpha)$ $=1775 \mathrm{keV}$ and $\left.\mathrm{E}\left({ }^{7} \mathrm{Li}\right)=1015 \mathrm{keV}\right)$. The light output from nuclear recoils in liquid scintillator is heavily quenched, to the level of $50-60 \mathrm{keV}_{e e}$ [6, 7], but, as will be shown, this is still detectable. The ability to detect neutron captures via scintillation from the nuclear recoil products makes relatively compact neutron detectors made from boron-loaded scintillator possible, as it is not necessary for the detector to be large enough to contain captureinduced gamma-rays.

Many applications of boron-loaded liquid scintillator neutron detectors make use of the delayed coincidence signals produced when energetic neutrons first produce recoil proton events during thermalization and are subsequently captured by ${ }^{10} \mathrm{~B}$. This is the basis of the "capture-gated neutron spectrometer" technique [8], and can be used to produce neutron detectors that can be operated in relatively high background environment: 1 . Development of neutron detectors based on this technique is ongoing (see, e.g., [9]), particularly for national security applications. In high efficiency neutron detectors, like the neutron vetoes described here, the coincidence technique cannot be exploited due to its lower overall efficiency (in the DarkSide-50-based simulations described below, only $\sim 80 \%$ of radiogenic neutrons produced a coincidence event in the boron-loaded scintillator).

TMB-loaded scintillators have also been investigated for use in large, low background particle detectors, as reported in [10, 11]. In particular, scintillator cocktails with TMB loading of up to $80 \%$, and with light output, optical attenuation, radio-purity, and scintillator stability properties suitable for a large, low-background neutrino experiment were identified in [10].

\section{Monte Carlo Modeling and Validation}

The Monte Carlo studies which constitute the bulk of this report were carried out using the Geant4 Toolkit (version 4.9.3) [12], via a flexible physics and geometry interface developed at Princeton University.

\footnotetext{
${ }^{1}$ Where the amount of light collected is high, gamma-ray interactions and neutron capture events in boron-loaded scintillator can also be distinguished using pulse shape analysis [7.
} 
The energy spectra used in simulating the radiogenic neutrons produced in different materials were determined by first calculating the individual spectra expected from $(\alpha, n)$ and fission processes due to ${ }^{235} \mathrm{U},{ }^{238} \mathrm{U}$, and ${ }^{232} \mathrm{Th}$ chain activities in each material using the SOURCES4A software package [13. These "component spectra" were then combined, using measured ratios of the different radioactive species, to give the total radiogenic neutron spectrum for each detector material. Cosmogenic neutrons were generated with the (depth dependent) approximate energy spectrum described in [14.

In what follows, "pure scintillator" is pseudocumene $\left(\mathrm{C}_{9} \mathrm{H}_{12}, \rho=0.876 \mathrm{~g} / \mathrm{cm}^{3}\right)$, while "boron loaded scintillator" is $50 \% \mathrm{w} / \mathrm{w}$ TMB in pseudocumene (this gives a composition of $62.3 \% \mathrm{C}, 23.1 \% \mathrm{O}, 9.4 \% \mathrm{H}$, and $5.2 \% \mathrm{~B}$ by mass) and is assumed to have a density equal to that of pseudocumene. The wavelength shifter necessary for the efficient optical performance of the scintillator, which is likely to be 2,5-diphenyloxazole (PPO) at the level of a few grams per litre in both boron-loaded and unloaded scintillator, will have a negligible effect on the overall neutron capture and is neglected in the simulation. All elements, including boron, are assumed to have natural isotopic abundances.

In order to confirm that the simulation provides a reasonable reproduction of neutron behavior, a number of "benchmark" comparisons have been made:

1. The simulated mean capture time for radiogenic neutrons in pseudocumene is $253 \pm 1 \mu \mathrm{s}$. This can be compared to the $256.0 \pm 0.4 \mu$ s neutron capture time observed by Borexino [15], which uses the same pseudocumene scintillator simulated here.

2. In the simulation, $99.1 \%$ of thermal neutrons captured in the boronloaded scintillator were captured by ${ }^{10} \mathrm{~B}$. Based on the ratio of the neutron capture cross-sections of the scintillator components (from [16]), one expects that $98.7 \pm 0.2 \%$ of thermal neutrons should be captured by ${ }^{10} \mathrm{~B}$ (at $19.9 \%{ }^{10} \mathrm{~B}$ abundance).

3. The fraction of neutron captures by ${ }^{10} \mathrm{~B}$ that produce ${ }^{7} \mathrm{Li}$ in the first excited state is $93.67 \pm 0.07 \%$ in the simulation, in good agreement with the expected value of $93.7 \%$ [5]

4. The mean free paths of neutrons of different energies deduced from the results of the simulation, as well as the mean free paths expected based on the cross sections in [5] are shown in Table 1.

These comparisons suggest that, although not perfect, neutron propagation and capture are reproduced reasonably well by the simulation code, and that 
the predictions of the neutron veto simulation might therefore be expected to provide reasonable predictions of the performance of an actual veto.

\begin{tabular}{|c|cc|cc|cc|}
\hline $\begin{array}{c}\text { Neutron Energy } \\
(\mathrm{MeV})\end{array}$ & \multicolumn{2}{|c|}{$\begin{array}{c}\text { MFP in Water } \\
(\mathrm{cm})\end{array}$} & \multicolumn{2}{c|}{$\begin{array}{c}\text { MFP in Pure } \\
\text { Scintillator }(\mathrm{cm})\end{array}$} & \multicolumn{2}{c|}{$\begin{array}{c}\text { MFP in Loaded } \\
\text { Scintillator }(\mathrm{cm})\end{array}$} \\
\hline & Sim. & XS & Sim. & XS & Sim. & XS \\
\hline 10 & 8.9 & 9.4 & 9.9 & 10.5 & 10.5 & 10.9 \\
20 & 12.8 & 11.5 & 13.3 & 11.8 & 13.6 & 12.3 \\
50 & 26.7 & 19.5 & 29.9 & 21.8 & 30.4 & - \\
100 & 40.9 & 38.0 & 45.3 & 43.3 & 46.6 & - \\
200 & 63.5 & - & 70.9 & - & 71.1 & - \\
\hline
\end{tabular}

Table 1: The mean free path (MFP) of neutrons of different energies in water, scintillator and boron-loaded scintillator, as simulated ("Sim.") and as calculated using the neutron interaction cross sections in 5 and the chemical composition of the veto ("XS," where data is available). In the Monte Carlo, only those interactions resulting in the creation of a secondary particle with more than $1 \mathrm{eV}$ of kinetic energy were directly recorded. Thus, some low energy interactions, low angle forward scattering for example, were excluded in the determination of the Monte Carlo MPFs; this could help to account for the longer interaction lengths in the Monte Carlo.

\section{Neutron Detection with Boron-Loaded Scintillator}

Using the Monte Carlo described above, we have simulated the thermalization and capture of radiogenic neutrons in pure and boron-loaded scintillators. Figure 1 shows the radial and time distributions of the simulated neutron captures. As can be seen, the addition of the boron reduces the mean neutron capture time by more than a factor of 100, from $253 \mu$ s to 2.3 $\mu \mathrm{s}$.

The distributions shown in Figure 1, while illustrative, do not correspond exactly to the distribution of veto event production in the scintillator. Two main factors contribute to this difference: first, capturing a neutron does not necessarily result in a veto signal at the point of capture, especially in pure scintillator. Instead, secondary particles, particularly gamma rays, which propagate some distance before depositing a detectable amount of energy in the scintillator, are often produced. Second, it is possible that the neutron deposits sufficient energy via nuclear recoils to produce a veto signal before being captured. As these recoils occur promptly, the recoil-induced veto signals can occur significantly earlier than the capture signals. 


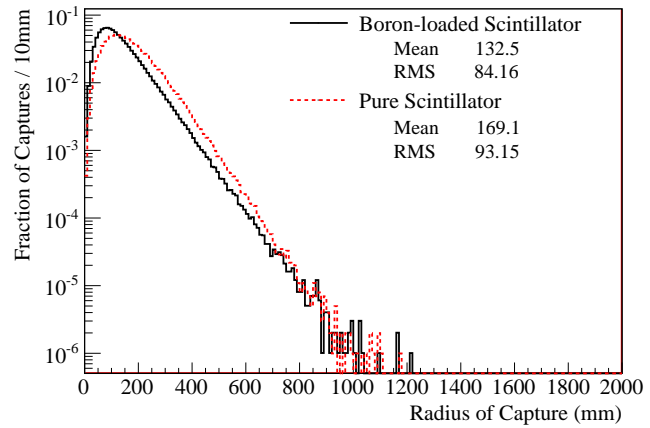

(a)

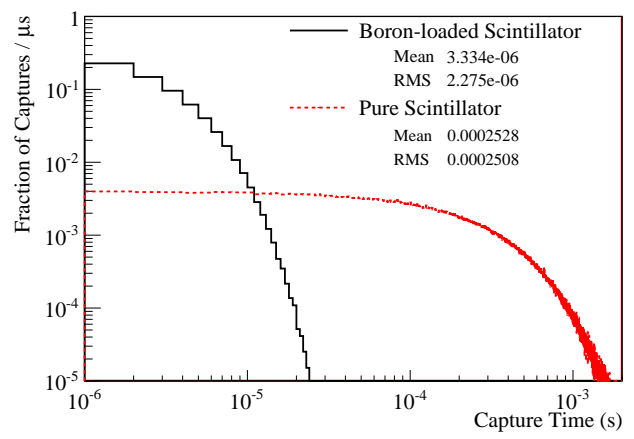

(b)

Figure 1: The simulated distributions in radius and time of the captures of centrally produced radiogenic neutrons in large, uniform volumes of boron-loaded and pure liquid scintillator. 
To investigate these effects, a "veto signal" was assumed to be generated in the simulation if $40 \mathrm{keV}_{e e}$ or more was deposited in the scintillator within any $1 \mu$ s time window. The quenching of heavy particles in the TMB-loaded scintillator was assumed to be identical to quenching in unloaded scintillator. Quenching for protons and alpha particles were treated separately following [17] and [18]; all heavier recoils were quenched as carbon [19, 17]. It might be expected that the use of quenching values from undiluted scintillator would underestimate the quenching effect in a diluted scintillator; however, the pure scintillator quenching values give $50 \mathrm{keV}_{e e}$ energy deposition in the scintillator from the recoil products of $n+{ }^{10} \mathrm{~B} \rightarrow \alpha+{ }^{7} \mathrm{Li}^{*}$, in good agreement with observations in TMB-loaded (and hence diluted) scintillator [6, 7].

Figure 2 shows the time distribution for the production of the first veto signal (if any) for each neutron event, and the detector radius necessary to contain the energy deposited in that trigger. As expected, in pure scintillator the spatial distribution of veto triggers is broader than the distribution of neutron captures because of gamma-ray propagation. For boron-loaded scintillator, by contrast, this broadening does not occur because the recoil daughters from neutron capture on ${ }^{10} \mathrm{~B}$ deposit sufficient energy at the site of capture to produce a veto signal. In fact, in boron-loaded scintillator the distribution of veto trigger production is narrower than the distribution of neutron captures, due to the generation of veto triggers by neutrons scattering prior to capture. Tables 2 and 3 contrast the radial and time windows necessary to contain, with different probabilities, the veto signal after the production of a radiogenic neutron in pure and boron-loaded scintillator. As can be seen, the addition of boron decreases the windows in both radius and time necessary to detect the veto neutrons with high probability.

\subsection{Other Loading Options}

We note that the reduction in the average capture time of neutrons in boron-loaded scintillator compared to pure scintillator could also be achieved by loading the scintillator with other isotopes possessing large neutron capture cross-sections, among them ${ }^{6} \mathrm{Li},{ }^{113} \mathrm{Cd}$, and ${ }^{157} \mathrm{Gd}$. Neutron capture on the latter isotopes is detected through the emission of gamma-rays, which raises the prospect that, as with neutron captures by protons, larger scintillator volumes would be necessary to contain the gamma rays. In our simulations, however, we find the neutron capture performance of a veto composed of scintillator loaded to $0.6 \%$ by weight with natural gadolinium offers very similar performance, in both capture time and radius of energy deposition, 


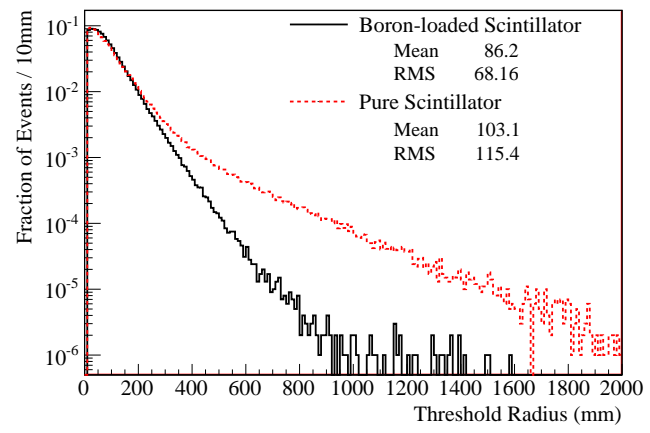

(a)

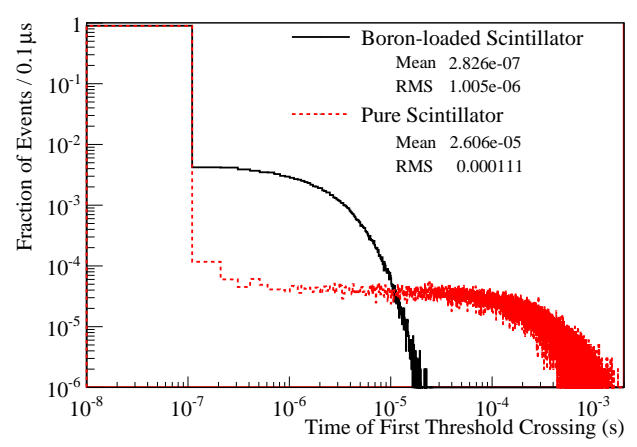

(b)

Figure 2: The radial positions and times at which the first veto trigger associated with each neutron event was generated.The $\mathrm{x}$-axis of (b) has been changed relative to Figure 1 to emphasize the prompt veto events created by nuclear recoils during neutron thermalization: such a prompt veto is produced by approximately $90 \%$ of neutrons in both pure and boronloaded scintillator. 


\begin{tabular}{ccc}
\hline \hline Containment Probability & $\begin{array}{c}\text { Pure Scintillator } \\
\text { Radius }(\mathrm{cm})\end{array}$ & $\begin{array}{c}\text { Loaded Scintillator } \\
\text { Radius }(\mathrm{cm})\end{array}$ \\
\hline $70 \%$ & 11.1 & 10.2 \\
$90 \%$ & 21.0 & 17.2 \\
$95 \%$ & 29.1 & 21.7 \\
$98 \%$ & 44.8 & 28.0 \\
$99 \%$ & 60.4 & 32.9 \\
$99.5 \%$ & 78.0 & 38.1 \\
$99.9 \%$ & 129.7 & 51.6 \\
$99.99 \%$ & - & 136.5 \\
\hline \hline
\end{tabular}

Table 2: The radius required to contain the scintillator veto signal with different probabilities.

\begin{tabular}{ccc}
\hline \hline Detection Efficiency & $\begin{array}{c}\text { Time in Pure } \\
\text { Scintillator }(\mu \mathrm{s})\end{array}$ & $\begin{array}{c}\text { Time in Loaded } \\
\text { Scintillator }(\mu \mathrm{s})\end{array}$ \\
\hline $70 \%$ & 0.08 & 0.08 \\
$90 \%$ & 7.8 & 0.1 \\
$95 \%$ & 185 & 1.7 \\
$98 \%$ & 421 & 3.8 \\
$99 \%$ & 603 & 5.4 \\
$99.5 \%$ & 788 & 7.0 \\
$99.9 \%$ & 1282 & 10.9 \\
$99.99 \%$ & - & 22.0 \\
\hline \hline
\end{tabular}

Table 3: The time interval after neutron production (and hence any prompt recoils in the WIMP detector) necessary to contain the veto signals with different probabilities. 
to the boron-loaded scintillator. The production of a cascade of gammarays, rather than a single photon, by neutron captures on ${ }^{157} \mathrm{Gd}$ seems to be responsible for the improved spatial performance of gadolinium relative to pure scintillator. Gadolinium loading offers the advantage that the majority of neutron captures on ${ }^{157} \mathrm{Gd}$ deposit more energy in the veto than do background gamma rays; this raises the possibility that neutron detection in Gd-loaded scintillator might be more robust against $\gamma$-ray backgrounds. In simulations including the inner WIMP detector from DarkSide-50 (described below), however, $6 \%$ of neutron events deposited less than $2 \mathrm{MeV}_{e e}$ in a veto composed of Gd-loaded scintillator and hence fall below the onset of the background from external gamma-rays, and $0.5 \%$ fell below $100 \mathrm{keV}$. Therefore, while gadolinium loading may in fact be preferable for a veto of moderately high efficiency (as it could be operated with less shielding), for very high-efficiency vetoes there is little, if any, difference between gadolinium and boron loading in the energy threshold required for the veto, and hence no difference in the tolerable rate of external background. We note also that TMB of acceptable radiopurity for the veto application was identified in [10], while some effort would likely be required to secure $\mathrm{Cd}$ or $\mathrm{Gd}$ with acceptable levels of contamination.

\section{A Boron-Loaded Scintillator Neutron Veto}

The discussion above suggests that a neutron veto efficiency greater than $99.9 \%$ could be obtained using a 1 meter thick boron-loaded liquid scintillator and an $11 \mu$ s time window. In actual operation, however, the veto will surround a detector of finite mass, which leads to the possibility that neutrons will be captured by components of the inner detector and hence will not be vetoed.

This effect has been investigated extensively in the context of the DarkSide50 experiment. DarkSide-50 is a proposed direct-detection dark matter experiment based on a 2-phase argon TPC, which will make use of argon depleted in ${ }^{39} \mathrm{Ar}$ from recently identified underground sources [20], and which will use the new ultra-low-background QUPID photon detectors [21]. These features, in addition to the use of a high-efficiency neutron veto, should not only give the $50 \mathrm{~kg}$ experiment a significant physics reach and the ability to make a convincing dark matter detection claim based on the observation of a few events, but will also give the relatively small experiment the potential to demonstrate directly that a larger detector of similar design could be 
operated in a background-free mode for several ton-years.

The DarkSide-50 geometry will essentially consist of a cylindrical active volume with equal diameter and height, contained in a fused silica inner vessel, with arrays of QUPIDs on the flat top and bottom faces. The vessel and QUPIDs are immersed in a (passive) liquid argon buffer inside a titanium (or low background stainless steel) cryostat 22]. For the purposes of this discussion of veto efficiency, the important features of DarkSide-50 are the masses of the individual detector components. These are listed in Table 4. as implemented in the simulation used here.

\begin{tabular}{ccc}
\hline \hline Component & Material & Mass (kg) \\
\hline Active Region & Depleted Argon & 52.7 \\
Inner Vessel + Photodetectors & Fused Silica & 25.4 \\
Passive Buffer & Depleted Argon & 74.1 \\
Cryostat + Inner Mechanics & Titanium & 78.6 \\
\hline \hline
\end{tabular}

Table 4: The masses of the different inner components of the DarkSide-50 detector, as simulated. Except for the active gas and liquid depleted argon, all components are passive absorbers of neutrons.

In DarkSide-50, which was explicitly designed for operation in a neutron veto, the materials used in the inner detector were chosen to minimize the number of neutrons lost to captures by inactive components, and hence to increase the veto efficiency. This is in contrast to dark matter detector designs which attempt to mitigate internal cosmogenic neutrons using (passive) internal neutron absorbers. We have studied the inclusion of such passive absorbers, and have found that the increase in veto efficiency associated with the removal of the absorbers significantly outweighed the loss of the relatively modest neutron reduction they afforded.

\subsection{Internal Radiogenic Neutrons}

For the DarkSide-50 detector described above, with the cryostat surrounded by a 1 meter thick boron-loaded liquid scintillator, radiogenic neutrons generated in the inner detector, specifically in the photosensors, produced veto events (as before, at least $40 \mathrm{keV}_{e e}$ of energy deposition within a $1 \mu \mathrm{s}$ window was required to produce a veto signal) with an efficiency of $99.78 \pm 0.01 \%$. The inefficiency due to neutron capture on the inner detector is small, not because neutrons are not captured by the inner detector (indeed, in this simulation about $21 \%$ of primary neutrons are captured by 
inner detector components), but because the majority of these inner detector captures result in the production of secondary particles, particularly gammarays, which are subsequently detected by the veto.

The potential for neutrons to thermally walk within the inner detector, the components of which have lower neutron capture cross sections than the boron-loaded scintillator, for significant periods of time means that the time distribution of neutron veto signals is longer with the detector present (see Table 5) than without (Table 3), and is no longer described at longer times by a single exponential (Figure 33). However, neither the veto times nor the veto efficiency appear to vary significantly between those neutron events which deposited energy in the active argon volume and those which did not.

\begin{tabular}{cc}
\hline \hline Detection Efficiency & Time Required $(\mu \mathrm{s})$ \\
\hline $70 \%$ & 0.08 \\
$90 \%$ & 0.37 \\
$95 \%$ & 2.3 \\
$98 \%$ & 5.5 \\
$99 \%$ & 9.3 \\
$99.5 \%$ & 21.5 \\
$99.8 \%$ & 57.7 \\
\hline \hline
\end{tabular}

Table 5: The time interval required after neutron production to contain the veto signals with different probabilities. The increase in the neutron capture time caused by the presence of the inner detector can be seen by comparing these values with those in in Table 3 .

The efficiency of the neutron veto might also be affected by the feedthroughs necessary to supply cryogenic cooling to the inner detector and to operate the photodetectors. To investigate this potential effect, a $10 \mathrm{~cm}$ diameter air-filled trunk was added to the simulation. The trunk passed through each of the inner vessel components (other than the active argon), and then out through the neutron veto. In the center of the veto, a $60 \mathrm{~cm}$ long right-angle "dog-leg" was added to the feedthrough, to eliminate straight paths of neutron escape. With the feedthrough in place, radiogenic neutrons generated in the photodetectors produced veto events with $99.75 \pm 0.02 \%$ efficiency, with no apparent effect on the time distribution of the veto events. Thus, it seems that the necessity of having a passive feedthrough within the neutron veto will not have a significant effect on the efficiency with which the neutron veto rejects internal radiogenic neutrons. 


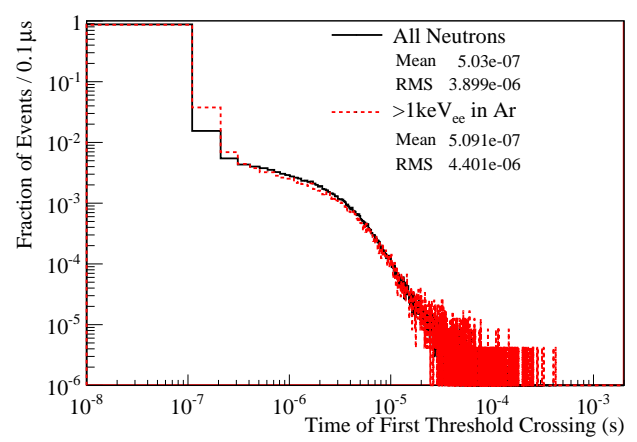

Figure 3: The simulated time distribution of veto events produced by radiogenic neutrons from the inner detector. As can be seen, the distribution of veto times is quite insensitive to whether or not the neutron deposited energy in the argon active volume of the inner detector. The fraction of all neutrons vetoed by a prompt recoil was $88 \%$ : for the neutrons which deposited some energy in the argon this value is $86 \%$.

In all, then, our simulations suggest that, with a $60 \mu$ s veto window, veto efficiencies higher than $99.7 \%$ could be obtained with a 1 meter thick neutron veto of boron-loaded scintillator around the DarkSide-50 detector, even after including the effects of neutron capture on the inner detector and a $10 \mathrm{~cm}$ diameter feedthrough in the veto. Such a veto could be expected to provide similar performance when used with other detectors of similar mass, provided that some care is taken to minimize neutron loss in the inner detector.

\subsection{External Radiogenic Neutrons}

In addition to its high detection efficiency for internally produced radiogenic neutrons, a boron-loaded scintillator veto is also an excellent shield against external radiogenic neutrons. Our simulations suggest that the fraction of external radiogenic neutrons that will penetrate the 1 meter neutron veto and produce a recoil in the active volume of DarkSide- 50 is $\lesssim 1 \times 10^{-7}$. It is expected that the residual external radiogenic events will be vetoed with an efficiency at least equal to the veto efficiency for internal radiogenic neutrons, giving an overall reduction in the rate of external radiogenic backgrounds of more than $10^{9}$, although we have not generated sufficient Monte Carlo statistics to confirm this.

\subsection{Cosmogenic Neutrons}

As dark matter experiments continue to increase in sensitivity, cosmogenic neutron backgrounds become a dominant background, even at rela- 
tively deep sites. These high-energy neutrons are quite penetrating, and are hence difficult to shield. Nonetheless, with relatively large shields, which are themselves instrumented as muon veto systems to reject cosmogenic backgrounds originating from within the shielding itself, cosmogenic backgrounds can be significantly reduced.

A liquid scintillator is ideal for use as a shield against external cosmogenic neutron ${ }^{2}$, as the scintillator has the potential to detect the cosmogenic neutrons, rather than simply attenuating them. This means that the mean free path (Table 1), rather than the attenuation length (Table 6), gives the appropriate scale for the reduction of the flux of external cosmogenic neutrons by a scintillator veto. In addition, the scintillator veto has the potential to detect cosmogenic neutrons both before and after they have interacted in the inner detector, as opposed to attenuation-based shielding which is effective on the incident particle only. Both the change from attenuation length to mean free path as the effective metric and the ability to detect the recoiling as well as the incident neutrons increase the effectiveness of the scintillator veto by approximately a factor of two relative to water shielding, meaning that, in aggregate, 1 meter of scintillator veto is about as effective as 4 meters of water shielding in reducing backgrounds due to external cosmogenic neutrons.

\begin{tabular}{cccc}
\hline \hline $\begin{array}{c}\text { Neutron Energy } \\
(\mathrm{MeV})\end{array}$ & $\begin{array}{c}\text { MDR in Water } \\
(\mathrm{cm})\end{array}$ & $\begin{array}{c}\text { MDR in Pure } \\
\text { Scintillator }(\mathrm{cm})\end{array}$ & $\begin{array}{c}\text { MDR in Loaded } \\
\text { Scintillator }(\mathrm{cm})\end{array}$ \\
\hline 10 & 22.2 & 26.3 & 27.5 \\
20 & 27.4 & 30.8 & 32.5 \\
50 & 54.8 & 60.5 & 62.7 \\
100 & 92.0 & 98.5 & 102.9 \\
200 & 145.7 & 168.2 & 170.4 \\
\hline \hline
\end{tabular}

Table 6: The mean position of the most distant nuclear recoil ("MDR") produced by neutrons of different energies (or any of their daughter particles). This metric provides an "effective attenuation length" appropriate for use in estimating neutron shielding necessary for direct-detection dark matter experiments.

\footnotetext{
${ }^{2}$ Note that the efficiency of the scintillator in vetoing those muons which pass through the scintillator directly is sufficiently high that internal cosmogenic backgrounds are strongly sub-dominant to the external cosmogenics, and as a result internal cosmogenics are not considered here.
} 
In order to obtain a quantitative estimate of the ability of the scintillator veto to reduce external cosmogenic neutrons, these were added to the DarkSide-50 simulation. The energy spectrum used was the parameterized spectrum given in [14] for cosmogenic neutron production at a depth of 3.1 km.w.e. (the effective depth of LNGS). Only primary neutrons with energies greater than $12 \mathrm{MeV}$ were simulated, as the lower energy neutrons are unlikely to penetrate the veto. We note that the energy spectrum of cosmogenic neutrons emerging from the rock walls will differ from the production spectrum somewhat due to rock attenuation - this effect was not considered here. Also, by generating single cosmogenic neutrons as the primary particles in our simulations, we neglect the possibility that other particles from the primary muon shower could enter the veto: this should cause us to underestimate, perhaps significantly, the efficiency of the veto against external cosmogenic neutrons.

Even in these very conservative simulations, however, a 1 meter thick scintillator veto reduced the number of recoil events in the active argon detector produced by cosmogenic neutrons with initial energies greater than $12 \mathrm{MeV}$ by about a factor of 40 (the number of recoil events were reduced by $\sim 25 \%$ from attenuation, with $\sim 97 \%$ of the remainder being vetoed) compared to the rate with no neutron shielding. We note that from the perspective of external cosmogenic neutrons, the factor of 40 reduction is equivalent to increasing the depth of the experiment by more than $2 \mathrm{~km}$.w.e.

For interest, we have simulated the suppression in external cosmogenic neutrons possible with thicker scintillator vetoes. As can be seen in Table 7 , cosmogenic neutron suppression continues to increase as the veto thickness increases.

\begin{tabular}{cc}
\hline $\begin{array}{c}\text { Veto Thickness } \\
(\mathrm{m})\end{array}$ & $\begin{array}{c}\text { Relative Recoil Rate in } \\
\text { DarkSide-50 }\end{array}$ \\
\hline 0 & 1.0 \\
1 & $(2.7 \pm 0.4) \times 10^{-2}$ \\
2 & $(2.4 \pm 0.6) \times 10^{-3}$ \\
3 & $(5 \pm 2) \times 10^{-4}$ \\
\hline \hline
\end{tabular}

Table 7: The factor by which the rate of external cosmogenic backgrounds is reduced (through a combination of attenuation and direct vetoing) by neutron vetoes of different thicknesses. 


\section{Veto Trigger Rates and Veto-Induced Dead Time}

The effective dead time produced by the veto in the inner detector depends on the event rate in the veto and the veto time window. For a $60 \mu \mathrm{s}$ veto window, which from Table 5 is necessary to maintain a very high veto efficiency, a $1 \%$ dead time would be produced by a veto rate of $168 \mathrm{~Hz}$, while a $10 \%$ dead time would be produced by a $1756 \mathrm{~Hz}$ veto rate.

The background rate in the veto will be due to external backgrounds, intrinsic backgrounds in the scintillator itself, background decays in the PMTs, and backgrounds from the inner detector. The latter will presumably be negligible on the scale of $100 \mathrm{~Hz}$, while the intrinsic rate in the scintillator will be dominated by $\left.{ }^{14} \mathrm{C}\right]^{3}$ which, at the $10^{-18}{ }^{14} \mathrm{C} /{ }^{12} \mathrm{C}$ ratio found in petrochemically-derived scintillator [10], would have a rate in the $11.5 \mathrm{~T}$ veto of 2-3 Hz.

While the background rate from PMT activity and external backgrounds will depend on the construction and location of the detector, it is possible to produce reasonable estimates of what these background rates could be. The total background decay rate in "low-background" 8" PMTs varies in the range of about 1-6 Hz, depending on the manufacturer. With 80 PMTs (see Section 7), the background rate would likely be 100-500 Hz. With the PMTs mounted so that their faces are flush with the outer wall of the veto (as assumed in the optical simulations), perhaps half of this activity would be detected by the veto.

External backgrounds will be dominated by gamma-rays produced in the rock surrounding the experiment. A reasonable estimate for the activity in "typical" rock is $15 \mathrm{~Bq} / \mathrm{kg}{ }^{238} \mathrm{U}+{ }^{232} \mathrm{Th}$ activity, and $300 \mathrm{~Bq} / \mathrm{kg}{ }^{40} \mathrm{~K}$ activity (there is, of course, significant variation in these activities between different sites). These correspond to fluxes of $\sim 2500 \mathrm{\gamma} / \mathrm{m}^{2} / \mathrm{s}$ for the 2.2 and $2.6 \mathrm{MeV}$ $\mathrm{U}$ and Th chain gamma-rays, and $\sim 42,000 \mathrm{\gamma} / \mathrm{m}^{2} / \mathrm{s}$ for the $1.4 \mathrm{MeV}$ gamma from K.

Taking DarkSide-50 as an example, the surface area of the veto will be about $31 \mathrm{~m}^{2}$, so for these levels of external activity we would expect $\sim 80,000$ $\mathrm{Hz}$ of incident $2.6 \mathrm{MeV}$ photons, and $\sim 1.3 \times 10^{6} \mathrm{~Hz}$ of $1.4 \mathrm{MeV}$ gammas ${ }^{4}$. As

\footnotetext{
${ }^{3}$ The reduction of $\mathrm{U}$ and $\mathrm{Th}$ backgrounds by distillation was demonstrated in [10] to be even more effective in TMB than in pseudocumene, so, as in 23, the rates of these "other" backgrounds should be small compared to ${ }^{14} \mathrm{C}$.

${ }^{4}$ We actually plan to deploy DarkSide-50 within the CTF water tank, an $11 \mathrm{~m}$ diameter
} 
these rates are too high for effective veto operation, the gamma-rays must be attenuated. We have explored relatively inexpensive scrap steel from decommissioned ships as a passive shield. The simulated attenuation factors for 2.6 and $1.4 \mathrm{MeV}$ gammas as a function of steel thickness are given in Table 8. For the rate of external gamma-rays described above, about $25 \mathrm{~cm}$ of steel would be sufficient to reduce the rate of external gamma-ray interactions in the veto to $\sim 150 \mathrm{~Hz}$. It should be noted that at some underground sites with lower gamma background rates, as little as half this passive shielding would be required.

\begin{tabular}{ccc}
\hline \hline $\begin{array}{c}\text { Steel Thickness } \\
(\mathrm{cm})\end{array}$ & $\begin{array}{c}\text { Survival Fraction } \\
\text { for } 2.6 \mathrm{MeV} \text { gammas }\end{array}$ & $\begin{array}{c}\text { Survival Fraction } \\
\text { for } 1.4 \mathrm{MeV} \text { gammas }\end{array}$ \\
\hline 0 & 1.0 & 1.0 \\
5 & 0.27 & 0.18 \\
10 & 0.061 & 0.027 \\
15 & 0.013 & $3.9 \times 10^{-3}$ \\
20 & $3.0 \times 10^{-3}$ & $5.9 \times 10^{-4}$ \\
25 & $6.4 \times 10^{-4}$ & $7.5 \times 10^{-5}$ \\
30 & $1.8 \times 10^{-4}$ & $1.4 \times 10^{-5}$ \\
35 & $1.7 \times 10^{-5}$ & $1.8 \times 10^{-6}$ \\
\hline \hline
\end{tabular}

Table 8: The effective attenuation of 2.6 and $1.4 \mathrm{MeV}$ gamma-rays in layers of steel of different thicknesses, as simulated using Geant4. The attenuation factor is calculated based on the number of events produced in the neutron veto by external gamma-rays of the relevant energies with different thicknesses of steel shielding.

An additional source of radioactive background would then be the steel itself. Measurements of the ship-breaking steel indicate that the $\mathrm{K}$ activity is less than $13 \mathrm{mBq} / \mathrm{kg}$, while the $\mathrm{U}$ and $\mathrm{Th}$ activity totals less than about $2.5 \mathrm{mBq} / \mathrm{kg}$. At these rates (and conservatively taking all $\mathrm{U}$ and $\mathrm{Th}$ decays to produce $2.6 \mathrm{MeV}$ gamma-rays), the simulation predicts a rate of about 65 $\mathrm{Hz}$ in the veto from activity in the steel.

A final source of veto-induced dead time in the inner detector will be random triggers produced by dark rate pile-up. The 80 8" PMTs that we assume will be used to instrument the veto can be reasonably assumed to

x 10 m high water tank at LNGS, which will significantly attenuate external backgrounds. Here we discuss a solution which might be more generally useful in other applications of a neutron veto. 
have a $1 \mathrm{kHz}$ dark rate. Requiring a coincidence between at least three PMTs within a $1 \mu$ s veto trigger window, and assuming that the dark hits are randomly distributed in time, the rate of random veto triggers can be calculated to be $80 \mathrm{~Hz}$.

Although the trigger rates induced in the veto by the different sources discussed above will vary depending on the location of the detector and the construction of the veto, we have nonetheless produced reasonable estimates for the rates in a "typical" installation. As shown in Table 9, this "typical" rate would be quite acceptable, with the inner detector incurring a dead time of about $3 \%$ from the veto.

\begin{tabular}{lc}
\hline \hline Background Source & Veto Rate $(\mathrm{Hz})$ \\
\hline \hline Inner Detector & $<1$ \\
Scintillator Background & 3 \\
PMTs & 200 \\
External Backgrounds with $25 \mathrm{~cm}$ steel & 150 \\
Steel Backgrounds & 65 \\
Random Veto Triggers $(1 \mathrm{kHz}$ dark rate) & 80 \\
\hline Total Veto Rate & 498 \\
\hline \hline
\end{tabular}

Table 9: The estimated rate of background events in the veto for a "typical" underground installation, with $25 \mathrm{~cm}$ of passive steel shielding. This total veto rate corresponds to a dead time of about $3 \%$ in the argon detector.

\section{Veto Optical Efficiency}

The $40 \mathrm{keV}_{e e}$ detection threshold in the veto assumed in the earlier analysis was chosen so as to include the $\left(\approx 50 \mathrm{keV}_{e e}\right)$ energy deposition by the recoil daughters after neutron capture on ${ }^{10} \mathrm{~B}$. As can be seen in Figure 4 , fewer than $5 \%$ of veto triggers deposit less than $100 \mathrm{keV}_{e e}$. Nevertheless, it is important that the veto be able to reliably detect events at the threshold level in order to maintain the very high veto efficiency.

The chief concern in detecting such low energy events, of course, is the collection of a sufficient number of photons from each event to identify the neutron capture. We have performed a baseline study of the optical efficiency of the scintillator veto if it were instrumented with 80 standard 8" PMTs evenly distributed over the outer (curved) side of the veto cylinder, with no PMTs on the top and bottom. A rather crude optical simulation, 


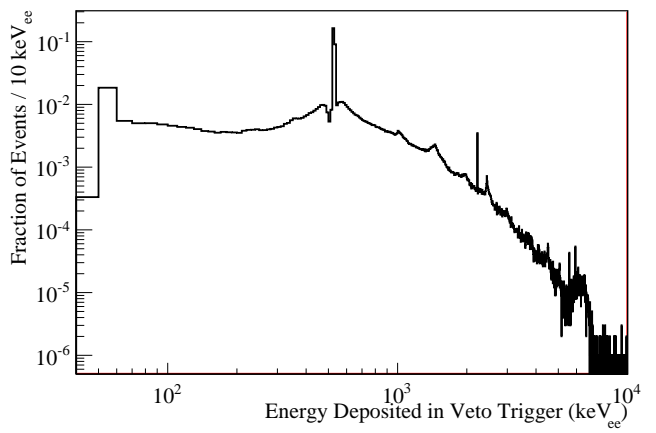

Figure 4: The energy spectrum of veto triggers generated by radiogenic neutrons. In each case the energy is deposited within a $1 \mu \mathrm{s}$ window; if more than one veto trigger was generated by a neutron event, only the earliest is plotted. The DarkSide-50 inner detector was present in this simulation - gamma lines from neutron capture on the inner detector components, as well as energy depositions from neutron scattering and capture within the veto itself can be seen in the spectrum.

independent of the Geant4 based physics simulation, was written to study the light collection efficiency of the veto in such a configuration. The model assumed:

1. A $20 \%$ quantum efficiency for the 8" PMTs. Photons that hit a PMT and were not detected were assumed to have a $20 \%$ probability of being reflected back into the veto.

2. A $95 \%$ reflection probability on all surfaces other than the PMTs (i.e. the inside walls of the veto tank and the outside of the DarkSide-50 cryostat).

3. An average scintillator light output of 6,000 photons $/ \mathrm{MeV}_{e e}$. The statistical fluctuation in the light output was assumed to be Gaussian, with a width of $\sqrt{N}$.

4. A $5 \mathrm{~m}$ optical absorption length in the scintillator, with no probability of re-emission.

5. A $2 \mathrm{~m}$ optical scattering length.

Each of these assumptions is extremely conservative:

1. 8" PMTs with quantum efficiencies $>30 \%$ are currently commercially available.

2. Both Spectralon PTFE and 3M Vikuiti foils, which are commercially 
available, have reflectances in excess of $95 \%$ for the relevant wavelengths.

3. Carefully prepared pseudocumene scintillator has typical light output of $\sim 12,000$ photons $/ \mathrm{MeV}_{e e}$ [24], and we have assumed that light output scales with TMB dilution. It is known, however, that the light output of scintillator with an optically inert dilutant, like TMB, typically decreases more slowly than the concentration!5. 80\% (rather than 50\%) TMB-loaded scintillator with a light output of 6000 photons/MeV was reported in [10].

4. Scattering and absorption lengths in TMB loaded scintillator greater than $10 \mathrm{~m}$ are reported in [10].

Figure 5 shows the simulated distributions of the total number of collected photoelectrons and the number of PMTs which detected at least one photoelectron when events of exactly $40 \mathrm{keV}_{e e}$ were simulated uniformly distributed throughout the veto volume. As discussed in Section 6, in order to maintain a reasonable rate of random triggers, a coincidence of 3 PMT hits would be required to trigger a veto event. As can be seen, even with the very conservative assumptions about the optical performance of the veto discussed above, in this simulation about $98 \%$ of $40 \mathrm{keV}_{e e}$ events produce a veto trigger. Given that events below $100 \mathrm{keV}_{e e}$ constitute less than $5 \%$ of the total, the overall fraction of neutron-induced backgrounds missed due to veto trigger inefficiency can therefore be expected to be significantly less than $0.1 \%$.

\section{Conclusions}

Neutron vetoes with very high efficiencies can be produced for direct detection dark matter experiments by surrounding the WIMP detector with a layer of liquid scintillator. Even after considering neutron loss in the inner detector and veto inefficiency due to feedthrough connections, our simulations suggest that a 1 meter thick veto can provide greater than a $99.5 \%$ efficiency for rejecting background events due to internal radiogenic neutrons, while reducing the background from external cosmogenic neutrons by more than $95 \%$. Loading ${ }^{10} \mathrm{~B}$, or another isotope with a high neutron capture cross

\footnotetext{
${ }^{5}$ For example, the KamLAND scintillator, which consists of pseudocumene diluted to $20 \%$ in dodecane, has a light output at least $\sim 80 \%$ that of pure PC 25.
} 


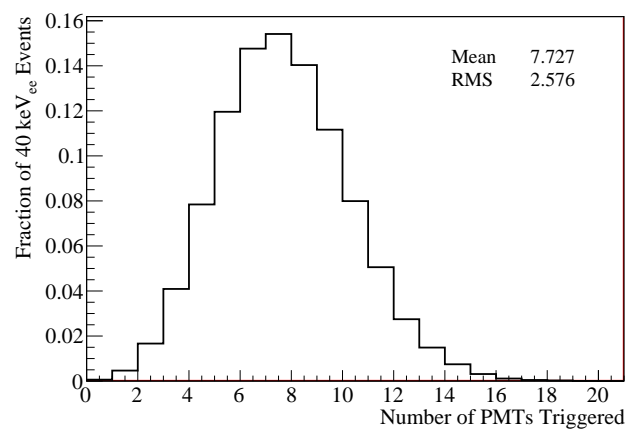

(a)

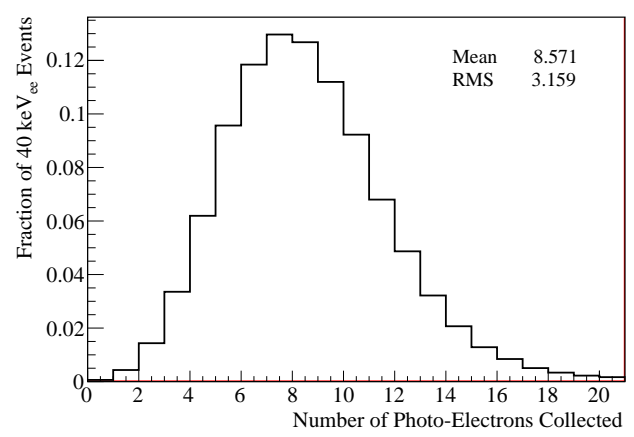

(b)

Figure 5: The distributions of (a) the number of PMTs which detected at least one photoelectron and (b) the total number of collected photoelectrons in simulations of 40 $\mathrm{keV}_{e e}$ events uniformly distributed through the neutron veto. 
section, into the scintillator makes the veto practical by greatly reducing the neutron capture time. This reduces the veto window necessary for high veto efficiency sufficiently so that conventional PMTs can be used in the veto, and typical underground background rates can be tolerated with relatively little external shielding.

\section{Acknowledgements}

The authors would like to thank the other members of the DarkSide collaboration and the members of the CDMS group at FNAL for interesting

and productive discussions about the practical aspects of the deployment of liquid scintillator neutron vetoes in dark matter experiments.

\section{References}

[1] R. Acciarri, et al., J. Phys.: Conference Series 203 (2010) 012006.

[2] D. Y. Akimov, et al., Astropart. Phys. 34 (2010) 151-63.

[3] C. Muehlhause, G. Thomas, Phys. Rev. 85 (1952) 926.

[4] L. Bollinger, G. Thomas, Rev. Sci. Instrum. 28 (1957) 489.

[5] M. Chadwick, et al., Nucl. Data Sheets 107 (2006) 2931-3060. Accessed via http://t2.lanl.gov/ and www.nndc.bnl.gov.

[6] L. Greenwood, N. Chellew, Rev. Sci. Instrum. 50(4) (1979) 466-71.

[7] S. Wang, et al., Nucl. Instrum. Meth. A 432 (1999) 111-21.

[8] G. Knoll, Radiation Detection and Measurement, John Wiley \& Sons, Inc., Hoboken, N.J., third edition, pp. 570.

[9] L. Swiderski, et al., in: Nuclear Science Symposium Conference Record, 2007. NSS '07. IEEE, volume 2, pp. 1389-95.

[10] G. Bellini, et al., Borexino at Gran Sasso. Proposal for a real time detector for low energy solar neutrinos: Volume 1, 1991.

[11] C. L. Cowan, et al., Phys. Rev. 90 (1953) 493-4.

[12] S. Agostinelli, et al., Nucl. Instrum. Meth. A 506 (2003) 250 - 303. 
[13] W. Wilson, et al., Progress in Nuclear Energy 51 (2009) 608 -13.

[14] D. Mei, A. Hime, Phys. Rev. D 73 (2006) 053004.

[15] M. Pallavicini, Recent Results from Borexino, 2010. Presentation at NOW2010.

[16] K. Shibata, et al., J. Nucl. Sci. Technol. 39 (2002) 1125. Accessed via http://www.ndc.jaea.go.jp/jendl/j33/j33.html.

[17] J. Hong, et al., Astropart. Phys. 16 (2002) 333 -8.

[18] F. Arneodo, et al., Nucl. Instrum. Meth. A 418 (1998) 285 -99.

[19] M. Steuer, B. Wenzel, Nucl. Instrum. Meth. 33 (1965) 131 -5.

[20] D. Acosta-Kane, et al., Nucl. Instrum. Methods A 587 (2008) 46-51.

[21] A. Fukasawa, et al., Nucl. Instrum. Meth. A: 623 (2010) 270 -2.

[22] D. Alton, et al., DarkSide: A Depleted Argon Dark Matter Search, 2010. Available http://www.fnal.gov/ directorate/program_planning/Nov2009PACPublic/DarkSideProposal.pdf.

[23] C. Arpesella, et al., Phys. Rev. Lett. 101 (2008) 091302.

[24] F. Elisei, et al., Nucl. Instrum. Meth. A 400 (1997) 53 - 68.

[25] F. Suekane, et al., in: KEK Proceedings: KEK-RCNP International School and Mini workshop for Scintillating Crystals and their Applications in Particle and Nuclear Physics, volume 4, pp. 279-90. An anthracene light output of 16,600 photons/MeV was assumed in interpreting this result. 